Document downloaded from:

http://hdl.handle.net/10251/60276

This paper must be cited as:

Guerrero, F.; Santonja, F.; Villanueva Micó, RJ. (2013). Solving a model for the evolution of smoking habit in Spain with homotopy analysis method. Nonlinear Analysis: Real World Applications. 14(1):549-558. doi:10.1016/j.nonrwa.2012.07.015.

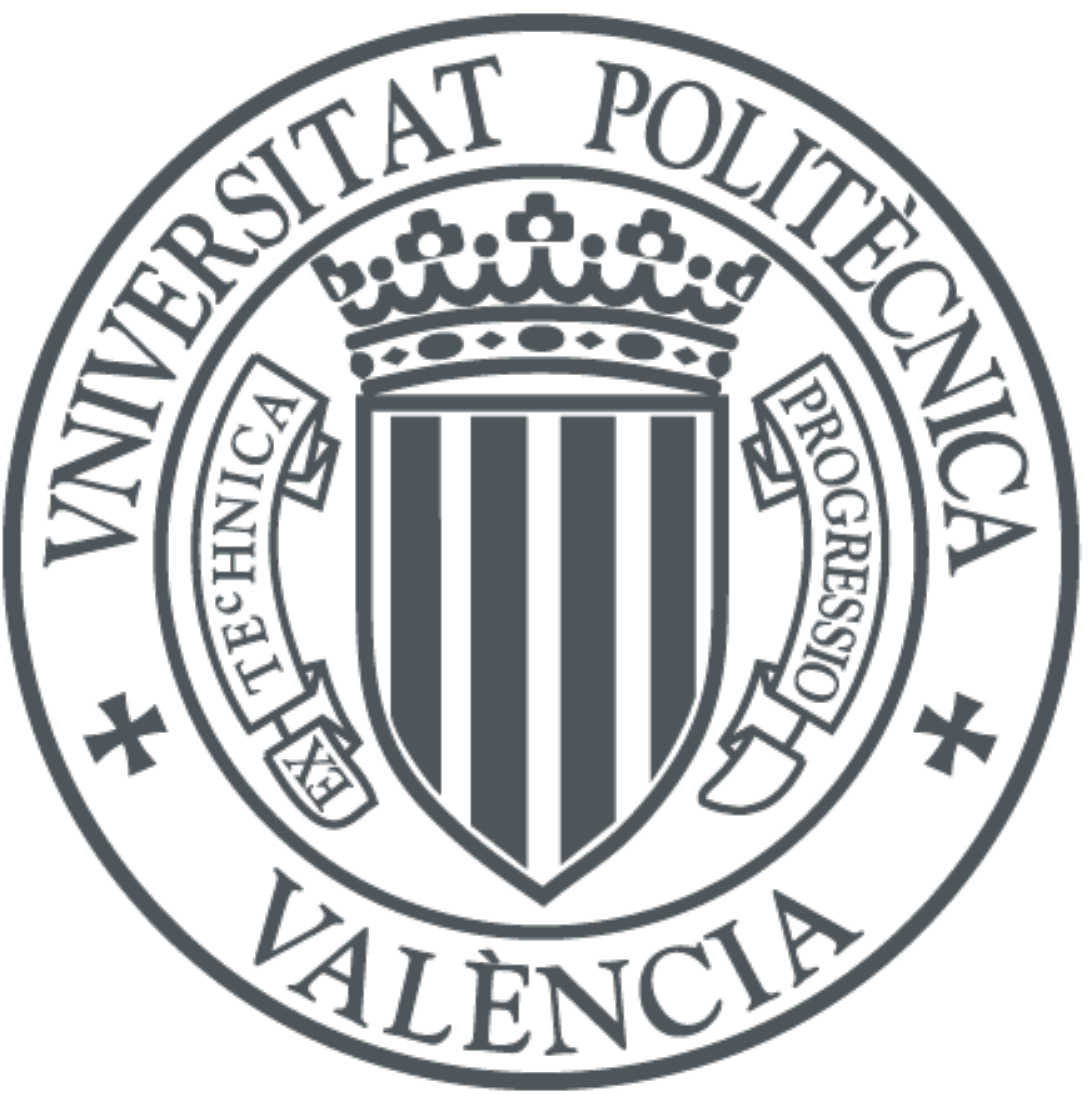

The final publication is available at

http://dx.doi.org/10.1016/j.nonrwa.2012.07.015

Copyright Elsevier

Additional Information 


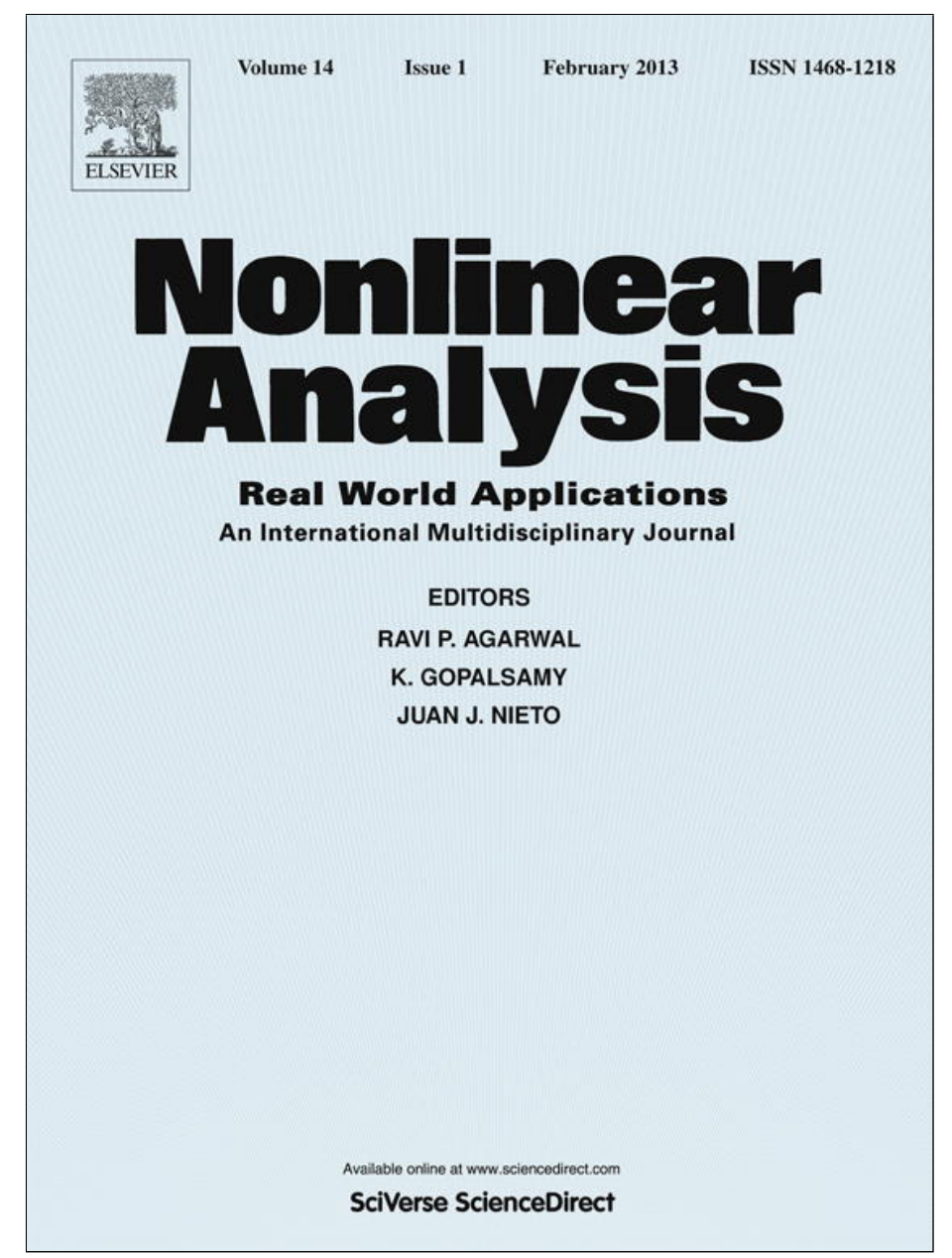

This article appeared in a journal published by Elsevier. The attached copy is furnished to the author for internal non-commercial research and education use, including for instruction at the authors institution and sharing with colleagues.

Other uses, including reproduction and distribution, or selling or licensing copies, or posting to personal, institutional or third party websites are prohibited.

In most cases authors are permitted to post their version of the article (e.g. in Word or Tex form) to their personal website or institutional repository. Authors requiring further information regarding Elsevier's archiving and manuscript policies are encouraged to visit:

http://www.elsevier.com/copyright 


\title{
Solving a model for the evolution of smoking habit in Spain with homotopy analysis method
}

\author{
F. Guerrero ${ }^{\text {a,* }}$, F.J. Santonja ${ }^{\text {b }}$, R.J. Villanueva ${ }^{c}$ \\ a Departamento de Matemática Aplicada, Universidad de Valencia, Av. Dr. Moliner, 50; 46100-Burjassot-Valencia, Spain \\ ${ }^{\mathrm{b}}$ Departamento de Estadística e Investigación Operativa, Universidad de Valencia, Spain \\ ${ }^{\mathrm{c}}$ Instituto de Matemática Multidisciplinar, Universidad Politecnica de Valencia, Spain
}

\section{A R T I C L E I N F O}

\section{Article history:}

Received 25 May 2012

Accepted 12 July 2012

\section{Keywords:}

Dynamic model

Epidemic model

Homotopy analysis method

Homotopy-Padé technique

Smoking model

\begin{abstract}
A B S T R A C T
We obtain an approximated analytical solution for a dynamic model for the prevalence of the smoking habit in a constant population but with equal and different from zero birth and death rates. This model has been successfully used to explain the evolution of the smoking habit in Spain. By means of the Homotopy Analysis Method, we obtain an analytic expression in powers of time $t$ which reproduces the correct solution for a certain range of time. To enlarge the domain of convergence we have applied the so-called optimal convergence-control parameter technique and the homotopy-Padé technique. We present and discuss graphical results for our solutions.
\end{abstract}

(c) 2012 Elsevier Ltd. All rights reserved.

\section{Introduction}

Epidemic models have been widely used to study epidemiological processes such as the spread of infectious diseases. This kind of model has also been used for the spread of social habits, such as the smoking habit [1], cocaine consumption [2], alcohol consumption [3] or obesity epidemics [4].

These models consist of a system of nonlinear ordinary differential equations that can be easily solved numerically by any standard numerical method. However, these integration algorithms give approximate solutions only at discrete points and they may also give rise to numerical instabilities such as oscillations, solutions that do not correspond to the solution of the original system of nonlinear ODEs, false equilibrium states, etc. [5].

To avoid this, we are interested in obtaining a continuous solution and this can be done by means of the Homotopy Analysis Method (HAM) initially developed by Liao [6,7].

In the past, HAM has been successfully used to solve many problems in science and engineering [8-15], and also in epidemic models such as SIR [16] and SIS models [17].

The main objective of this paper is to use HAM to obtain an analytical solution for the model for the spread of the smoking habit in Spain presented in [1]. This model has been used successfully to explain the evolution of the prevalence of smoking in Spain. According to this, we are using real data for the initial values and for the parameters of the system. We assume a constant population with birth rate and death rate equal and different from zero. This fact makes the total number of individuals constant, but those individuals are continuously renewed.

We obtain an analytic expression in powers of time $t$ which reproduces the correct solution for a certain range of time. The HAM provides a family of solutions depending on an auxiliary parameter $h$. The value of this parameter controls the

\footnotetext{
* Corresponding author. Tel.: +34 963543232; fax: +34 963543922 .

E-mail address: guecor@uv.es (F. Guerrero).
} 
convergence region of the solution. To determine the best value of $h$ we have used the so-called optimal convergencecontrol parameter technique [10,11]. Finally, to increase further the convergence domain of the analytical approximation we have applied the homotopy-Padé technique [6] with good results.

\section{The mathematical model}

The following model has been used successfully to predict the evolution of the prevalence of smoking in Spain and to evaluate the impact of the Spanish smoke-free law of 2006 [1].

We assume the population consists of four types of individuals, whose proportions are denoted by $n$ (non-smokers), $s$ (normal smokers), $c$ (excessive smokers) and $e$ (ex-smokers). All of them are functions of time.

$n(t)$ is the proportion of the total population who has never smoked, $s(t)$ is the proportion of people who smoke less than 20 cigarettes per day, $c(t)$ is the proportion of individuals who smoke more than 20 cigarettes per day and $e(t)$ is the proportion of ex-smokers.

The dynamics of the smokers in Spain is given by the following system of differential equations (see [1] for details)

$$
\begin{aligned}
& n^{\prime}(t)=\mu(1-n(t))-\beta n(t)(s(t)+c(t)), \\
& s^{\prime}(t)=\beta n(t)(s(t)+c(t))+\rho e(t)+\alpha c(t)-(\gamma+\lambda+\mu) s(t), \\
& c^{\prime}(t)=\gamma s(t)-(\alpha+\delta+\mu) c(t), \\
& e^{\prime}(t)=\lambda s(t)+\delta c(t)-(\rho+\mu) e(t) .
\end{aligned}
$$

The parameters of the model assumed constant are:

- $\mu$, birth rate in Spain.

- $\beta$, transmission rate due to social pressure to adopt smoking habit.

- $\rho$, rate at which ex-smokers return to smoking.

- $\alpha$, rate at which an excessive smoker becomes a normal smoker by decreasing the number of cigarettes per day.

- $\gamma$, rate at which normal smokers become excessive smokers by increasing the number of cigarettes per day.

- $\lambda$, rate at which normal smokers stop smoking.

- $\delta$, rate at which excessive smokers stop smoking.

Since the constant population has been normalized to unity, our variables satisfy that:

$$
n(t)+s(t)+c(t)+e(t)=1 .
$$

\section{The Homotopy Analysis Method (HAM)}

In this section the basic ideas of the HAM [6] are shown.

Consider

$$
N[y(t)]=0,
$$

where $N$ is any operator, $y(t)$ is the unknown solution of the system of differential equations that we want to solve and $t$ is the independent variable. We denote $y_{0}(t)$ the initial guess of the exact solution. $h \neq 0$ is an auxiliary parameter, $H(t) \neq 0$ is an auxiliary function, and $L$ an auxiliary operator that satisfies the property $L[y(t)]=0$ when $y(t)=0$.

Using $0<q<1$ as a parameter we construct the homotopy

$$
(1-q) L\left[\phi(t, q)-y_{0}(t)\right]-q h H(t) N[\phi(t, q)]=\hat{H}\left[\phi(t, q), y_{0}(t), H(t), h, q\right] .
$$

Setting the right hand side of (7) equal to zero, we obtain the zero-order deformation equation

$$
(1-q) L\left[\phi(t, q)-y_{0}(t)\right]=q h H(t) N[\phi(t, q)] .
$$

We can see that for $q=0$ the zero-order deformation equation becomes

$$
\phi(t, 0)=y_{0}(t)
$$

and when $q=1$, taking into account that $h \neq 0$ and $H(t) \neq 0$, the zero-order deformation equation is

$$
\phi(t, 1)=y(t) .
$$

Then, as the parameter $q$ increases from 0 to 1 , the function $\phi(t, q)$ changes continuously from the initial $y_{0}(t)$ to the exact solution $y(t)$. This type of continuous variation is called deformation in homotopy.

We expand $\phi(t, q)$ by Taylor's theorem in a power series of $q$

$$
\phi(t, q)=y_{0}(t)+\sum_{m=1}^{\infty} y_{m}(t) q^{m}
$$


where

$$
y_{m}(t)=\left.\frac{1}{m !} \frac{\partial^{m} \phi(t, q)}{\partial q^{m}}\right|_{q=0} .
$$

Assuming that $y_{0}(t)$, the operator $L$, the parameter $h$ and the function $H(t)$ are chosen such that

1. $\phi(t, q)$ exists for all $0 \leq q \leq 1$,

2. $y_{m}(t)$ exists for $m=1,2, \ldots$, and

3. the power series (11) of $\phi(t, q)$ is convergent for $q=1$,

we obtain the solution series

$$
\phi(t, 1)=y_{0}(t)+\sum_{m=1}^{\infty} y_{m}(t) .
$$

Differentiating the zero-order deformation equation (8) $m$ times with respective to $q$, dividing by $m$ ! and setting $q=0$, we get the $m$ th-order deformation equation

$$
L\left[y_{m}(t)-\chi_{m} y_{m-1}(t)\right]=h H(t) \mathcal{R}_{m}\left(y_{m-1}(t)\right)
$$

where

$$
\mathcal{R}_{m}\left(y_{m-1}(t)\right)=\frac{1}{(m-1) !} \frac{\partial^{m-1} N[\phi(t, q)]}{\partial q^{m-1}}
$$

with $\chi_{m}=0$ for $m \leq 1$ and $\chi_{m}=1$ for $m>1$.

Eq. (15) allows us to obtain $y_{m}(t)$ from $y_{m-1}(t)$. This means that solving Eq. (15) we are going to obtain $y_{1}(t), y_{2}(t), \ldots$ and so on.

\section{Solution of the smoking model by HAM}

To obtain the analytical approximation of the model of Eqs. (1)-(4), we use HAM has shown in [6,7].

First, we take the initial approximations of $n(t), s(t), c(t)$ and $e(t)$ as constant functions.

$$
n_{0}(t)=n_{0}, \quad s_{0}(t)=s_{0}, \quad c_{0}(t)=c_{0}, \quad e_{0}(t)=e_{0} .
$$

The HAM is based on a kind of continuous mappings

$$
n(t) \rightarrow \phi_{1}(t, q), \quad s(t) \rightarrow \phi_{2}(t, q), \quad c(t) \rightarrow \phi_{3}(t, q), \quad e(t) \rightarrow \phi_{4}(t, q),
$$

such that, as $q$ increases from 0 to $1, \phi_{i}(t, q)$ moves from the initial approximation to the exact solution.

Following [16], the auxiliary linear operators are defined by

$$
L_{i}\left[\phi_{i}(t, q)\right]=\frac{\partial \phi_{i}(t, q)}{\partial t}, \quad i=1,2,3, \ldots,
$$

satisfying that,

$$
L_{i}\left[C_{i}\right]=0
$$

for the integral constants $C_{i}$.

The non-linear operators $N_{i}$ are defined according to Eqs. (1)-(4),

$$
\begin{aligned}
& N_{1}\left[\phi_{i}(t, q)\right]=\frac{\partial \phi_{i}(t, q)}{\partial t}-\mu\left(1-\phi_{i}(t, q)\right)+\beta \phi_{i}(t, q)\left(\phi_{2}(t, q)+\phi_{3}(t, q)\right), \\
& N_{2}\left[\phi_{i}(t, q)\right]=\frac{\partial \phi_{i}(t, q)}{\partial t}-\beta \phi_{1}(t, q)\left(\phi_{i}(t, q)+\phi_{3}(t, q)\right)-\rho \phi_{4}(t, q)-\alpha \phi_{3}(t, q)+(\gamma+\lambda+\mu) \phi_{i}(t, q), \\
& N_{3}\left[\phi_{i}(t, q)\right]=\frac{\partial \phi_{i}(t, q)}{\partial t}-\gamma \phi_{2}(t, q)+(\alpha+\delta+\mu) \phi_{i}(t, q), \\
& N_{4}\left[\phi_{i}(t, q)\right]=\frac{\partial \phi_{i}(t, q)}{\partial t}-\lambda \phi_{2}(t, q)-\delta \phi_{3}(t, q)+(\rho+\mu) \phi_{i}(t, q) .
\end{aligned}
$$

According to zero-order deformation equation (8), a family of equations can be constructed,

$$
\begin{aligned}
& (1-q) L\left[\phi_{1}(t, q)-n_{0}(t)\right]=q h_{1} H_{1}(t) N_{1}\left[\phi_{1}(t, q)\right], \\
& (1-q) L\left[\phi_{2}(t, q)-s_{0}(t)\right]=q h_{2} H_{2}(t) N_{2}\left[\phi_{2}(t, q)\right], \\
& (1-q) L\left[\phi_{3}(t, q)-c_{0}(t)\right]=q h_{3} H_{3}(t) N_{3}\left[\phi_{3}(t, q)\right], \\
& (1-q) L\left[\phi_{4}(t, q)-e_{0}(t)\right]=q h_{4} H_{4}(t) N_{4}\left[\phi_{4}(t, q)\right],
\end{aligned}
$$

satisfying the initial conditions $\phi_{1}(0, q)=n_{0}, \phi_{2}(0, q)=s_{0}, \phi_{3}(0, q)=c_{0}$ and $\phi_{4}(0, q)=e_{0}$. 
Applying Eq. (13) to this case, we can write

$$
\begin{aligned}
& \phi_{1}(t, 1)=n_{0}(t)+\sum_{m=1}^{\infty} n_{m}(t), \\
& \phi_{2}(t, 1)=s_{0}(t)+\sum_{m=1}^{\infty} s_{m}(t), \\
& \phi_{3}(t, 1)=c_{0}(t)+\sum_{m=1}^{\infty} c_{m}(t), \\
& \phi_{4}(t, 1)=e_{0}(t)+\sum_{m=1}^{\infty} e_{m}(t),
\end{aligned}
$$

and

$$
\begin{aligned}
& n_{m}(t)=\left.\frac{1}{m !} \frac{\partial^{m} \phi_{1}(t, q)}{\partial q^{m}}\right|_{q=0} \\
& s_{m}(t)=\left.\frac{1}{m !} \frac{\partial^{m} \phi_{2}(t, q)}{\partial q^{m}}\right|_{q=0} \\
& c_{m}(t)=\left.\frac{1}{m !} \frac{\partial^{m} \phi_{3}(t, q)}{\partial q^{m}}\right|_{q=0} \\
& e_{m}(t)=\left.\frac{1}{m !} \frac{\partial^{m} \phi_{4}(t, q)}{\partial q^{m}}\right|_{q=0} .
\end{aligned}
$$

The $m$ th-order deformation equations (14) for this particular case are:

$$
\begin{aligned}
& L\left[n_{m}(t)-\chi_{m} n_{m-1}(t)\right]=h_{1} H_{1}(t) \mathcal{R}_{m}\left(n_{m-1}(t)\right) \\
& L\left[s_{m}(t)-\chi_{m} s_{m-1}(t)\right]=h_{2} H_{2}(t) \mathcal{R}_{m}\left(s_{m-1}(t)\right) \\
& L\left[c_{m}(t)-\chi_{m} c_{m-1}(t)\right]=h_{3} H_{3}(t) \mathcal{R}_{m}\left(c_{m-1}(t)\right) \\
& L\left[e_{m}(t)-\chi_{m} e_{m-1}(t)\right]=h_{4} H_{4}(t) \mathcal{R}_{m}\left(e_{m-1}(t)\right) \\
& n_{m}(0)=0, s_{m}(0)=0, c_{m}(0)=0 \text { and } e_{m}(0)=0 .
\end{aligned}
$$

Following [6] we set $H_{i}(t)=1$, for $i=1,2,3,4$. We also set $h_{1}=h_{2}=h_{3}=h_{4}=h$ so that $h$ will be the convergencecontrol parameter. Then, the $m$ th-order deformation equations for $m \geq 1$ are:

$$
\begin{aligned}
n_{m}(t)= & \chi_{m} n_{m-1}(t)+h \int_{0}^{t} d \tau\left[n_{m-1}^{\prime}(\tau)-\mu\left(s_{m-1}(\tau)+c_{m-1}(\tau)+e_{m-1}(\tau)\right)\right. \\
& \left.+\beta \sum_{k=0}^{m-1}\left(s_{k}(\tau)+c_{k}(\tau)\right) n_{m-1-k}(\tau)\right] \\
s_{m}(t)= & \chi_{m} s_{m-1}(t)+h \int_{0}^{t} d \tau\left[s_{m-1}^{\prime}(\tau)-\beta \sum_{k=0}^{m-1}\left(s_{k}(\tau)+c_{k}(\tau)\right) n_{m-1-k}(\tau)\right. \\
& \left.-\rho e_{m-1}(\tau)-\alpha c_{m-1}(\tau)+(\gamma+\lambda+\mu) s_{m-1}(\tau)\right] \\
c_{m}(t)= & \chi_{m} c_{m-1}(t)+h \int_{0}^{t} d \tau\left[c_{m-1}^{\prime}(\tau)-\gamma s_{m-1}(\tau)+(\alpha+\delta+\mu) c_{m-1}(\tau)\right] \\
e_{m}(t)= & \chi_{m} e_{m-1}(t)+h \int_{0}^{t} d \tau\left[e_{m-1}^{\prime}(\tau)-\lambda s_{m-1}(\tau)-\delta c_{m-1}(\tau)+(\rho+\mu) e_{m-1}(\tau)\right]
\end{aligned}
$$

With these formulae we are able to compute the functions $n_{m}(t), s_{m}(t), c_{m}(t)$ and $e_{m}(t)$. 


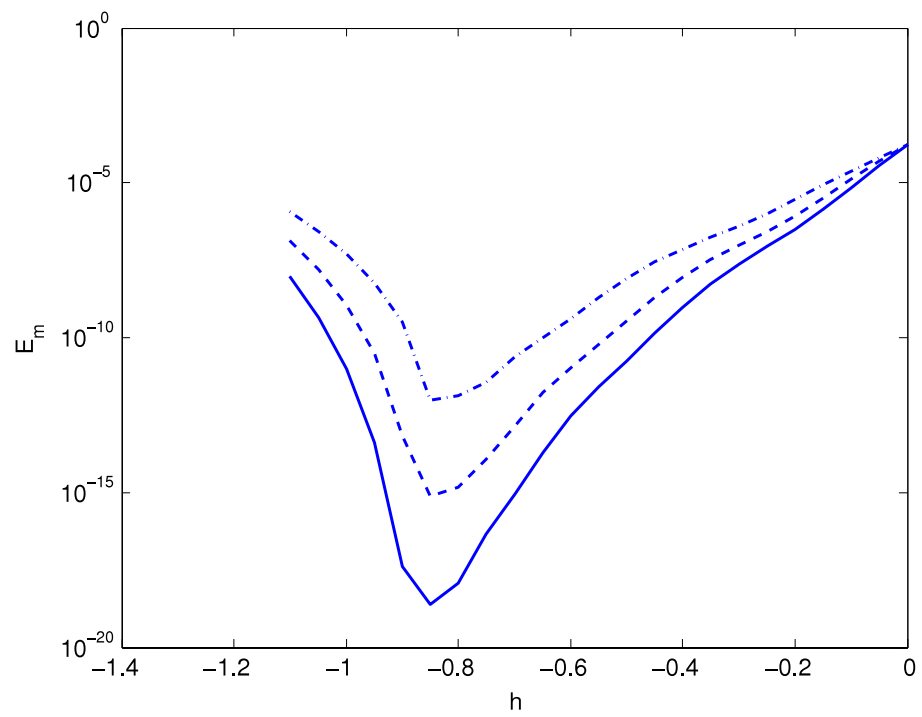

Fig. 1. Averaged residual error $E_{m}$ versus $h$. Dash-dotted line for $m=6$, dashed line for $m=8$ and solid line for $m=10$.

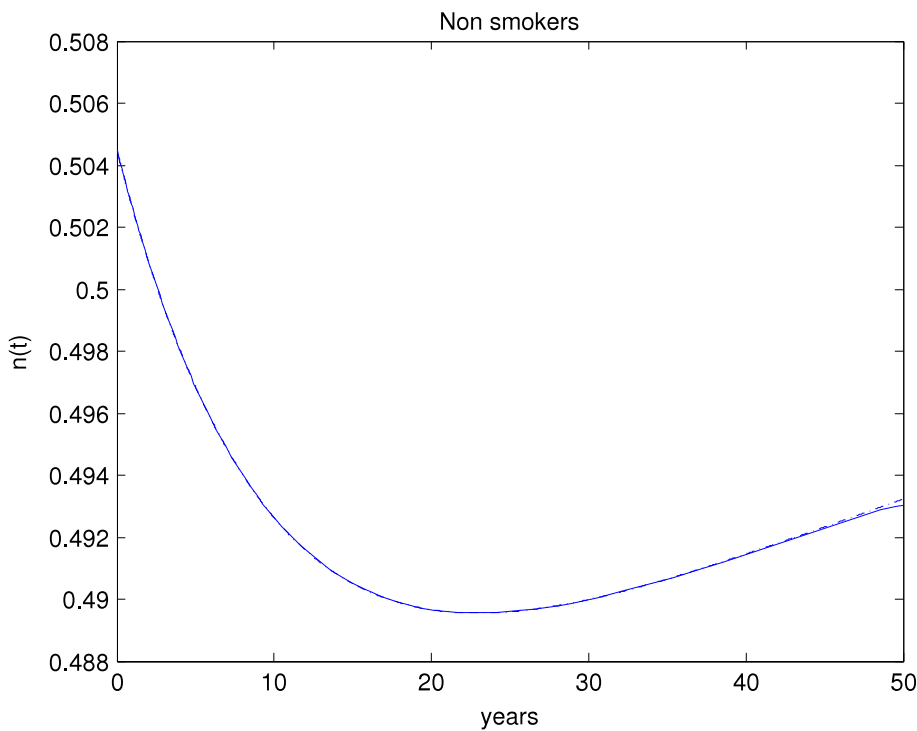

Fig. 2. Solution of HAM with 20 terms for $n(t)$ (solid line) and the exact solution (dash-dotted line). Note that one is on the other.

Table 1

Parameters of the model for the evolution of the prevalence of smoking in Spain [1].

\begin{tabular}{ll}
\hline Parameter & Value $\left(\right.$ year $\left.^{-1}\right)$ \\
\hline$\mu$ & 0.01 \\
$\rho$ & 0.0425 \\
$\beta$ & 0.0381 \\
$\alpha$ & 0.1244 \\
$\gamma$ & 0.1175 \\
$\lambda$ & 0.0498 \\
$\delta$ & 0.0498 \\
\hline
\end{tabular}

\section{Results}

Using Eqs. (28)-(31) and Eqs. (40)-(43) an analytical approximation for the solution of the smoking model is obtained. The values we use for the parameters of the model are shown in Table 1. These values have been estimated in [1] using real data for the Spanish population. 


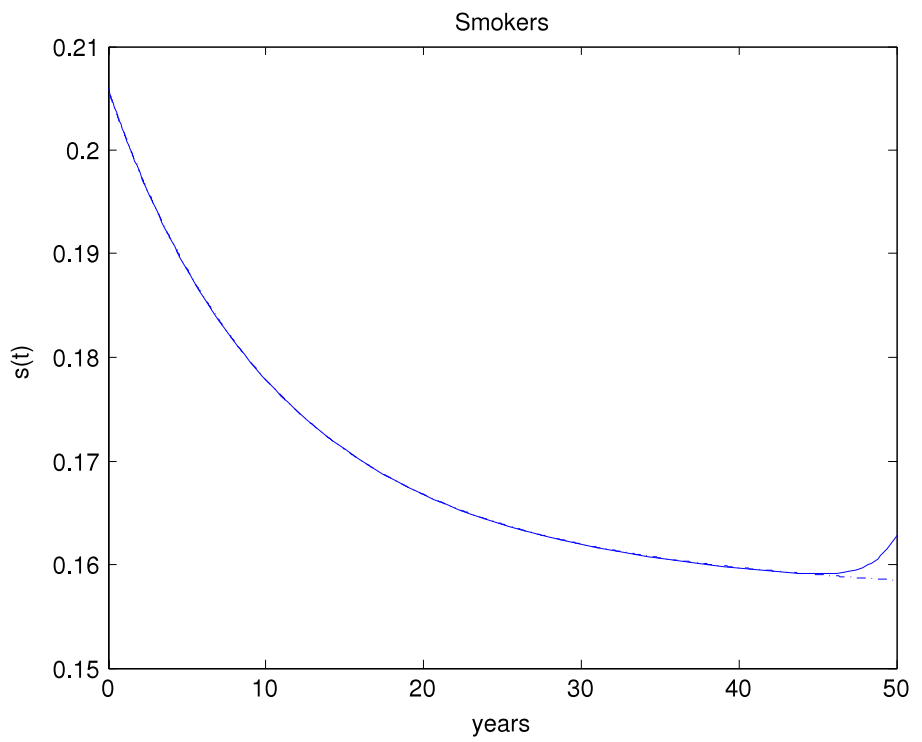

Fig. 3. Solution of HAM with 20 terms for $s(t)$ (solid line) and the exact solution (dash-dotted line). Differences appear for $t \geq 45$.

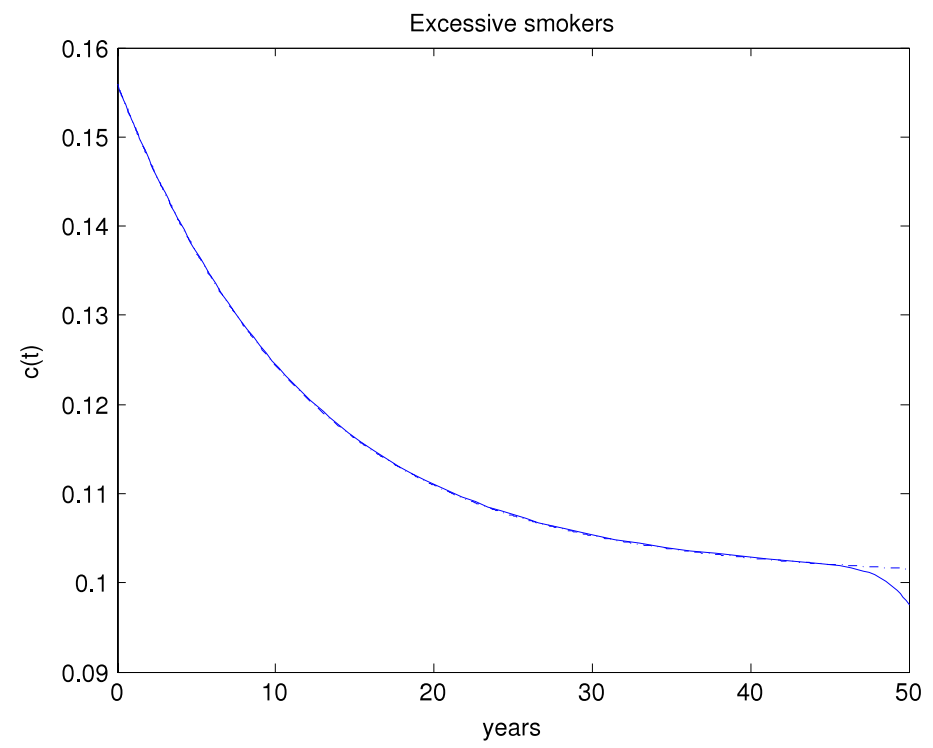

Fig. 4. Solution of HAM with 20 terms for $c(t)$ (solid line) and the exact solution (dash-dotted line). Differences appear for $t \geq 45$.

The initial values $n_{0}, s_{0}, c_{0}$ and $e_{0}$ are the real proportions for the subpopulations in Spain at the beginning of the period of time analyzed in [1].

$$
n_{0}=0.5045, \quad s_{0}=0.2059, \quad c_{0}=0.1559, \quad e_{0}=0.1337 .
$$

\subsection{Optimal convergence}

As we said above, parameter $h$ is called the convergence-control parameter. We have to determine the value of $h$ that provides the largest convergence domain for a given number $m$ of terms in the analytical approximation for the solution.

Following [10], we find out the so-called optimal convergence-control parameter. This technique establishes that the optimal value for $h$ is the one that minimizes the sum of the residual squares of the four Eqs. (1)-(4), given by:

$$
\begin{aligned}
E_{m}= & \frac{1}{K} \sum_{j=0}^{K}\left\{\left[N_{1}\left(\sum_{k=0}^{m} n_{k}(j \Delta t)\right)\right]^{2}+\left[N_{2}\left(\sum_{k=0}^{m} s_{k}(j \Delta t)\right)\right]^{2}\right. \\
& \left.+\left[N_{3}\left(\sum_{k=0}^{m} c_{k}(j \Delta t)\right)\right]^{2}+\left[N_{4}\left(\sum_{k=0}^{m} e_{k}(j \Delta t)\right)\right]^{2}\right\},
\end{aligned}
$$

where we have taken $K=20$ and $\Delta t=1$. $E_{m}$ is the so-called averaged residual error. 


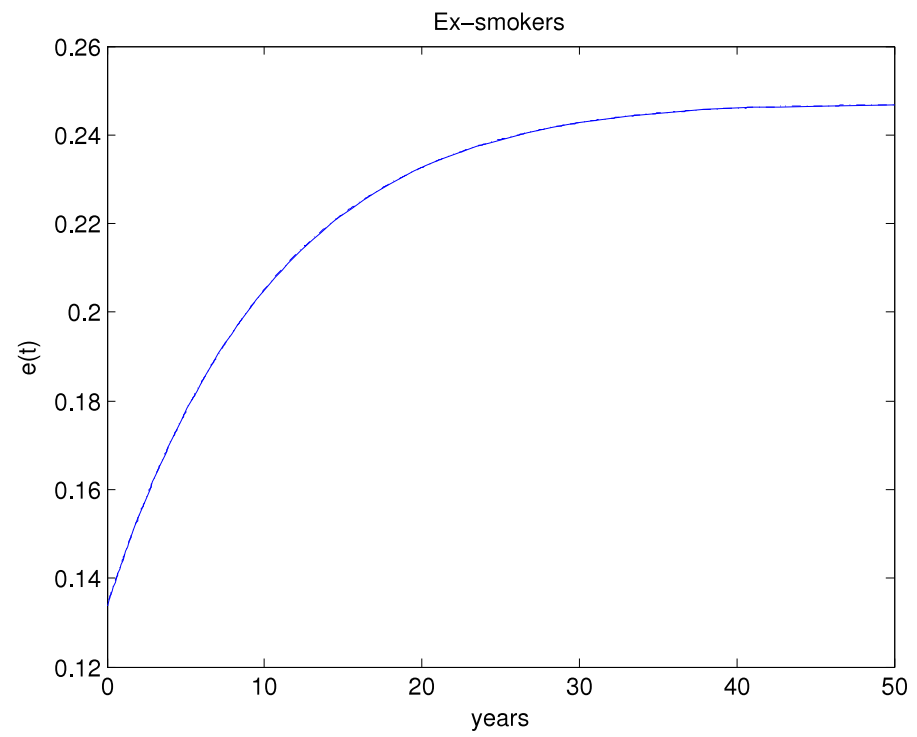

Fig. 5. Solution of HAM with 20 terms for $e(t)$ (solid line) and the exact solution (dash-dotted line).

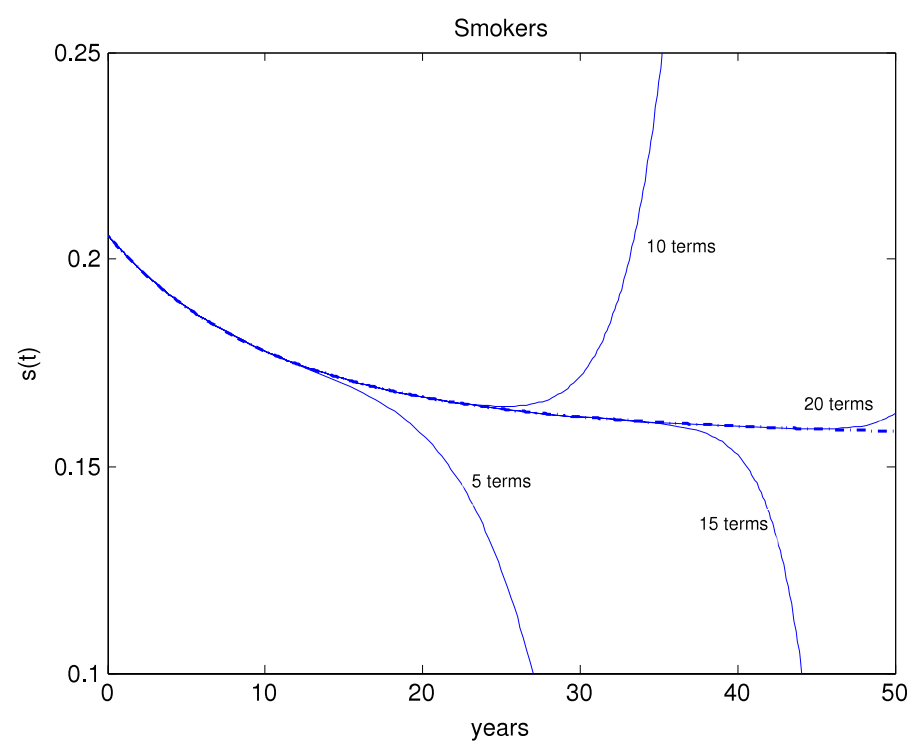

Fig. 6. Solutions of HAM with 5, 10, 15 and 20 terms for $s(t)$ (solid lines) and the exact solution (dash-dotted line). Note the improvement as the number of terms increases.

Fig. 1 shows the values of $E_{m}$ for different values of $h$ and for $m=6,8$ and 10 . We find out that the sum of residual squares is minimum for $h$ about -0.85 .

We present below ten terms approximations for $n(t), s(t), c(t)$ and $e(t)$ obtained for the optimal value $h=-0.85$

$$
\begin{aligned}
n(t)^{(10)}= & 0.5045-0.001999 t+0.00011026 t^{2}-3.68167 \cdot 10^{-6} t^{3}+9.6534 \cdot 10^{-8} t^{4}-2.2211 \cdot 10^{-9} t^{5} \\
& +4.7959 \cdot 10^{-11} t^{6}-9.8329 \cdot 10^{-13} t^{7}+1.7857 \cdot 10^{-14} t^{8}-2.4224 \cdot 10^{-16} t^{9}+1.7194 \cdot 10^{-18} t^{10} \\
s(t)^{(10)}= & 0.2059-0.004496 t+0.0002521 t^{2}-1.2627 \cdot 10^{-5} t^{3}+6.5927 \cdot 10^{-7} t^{4}-3.4315 \cdot 10^{-8} t^{5} \\
& +1.6277 \cdot 10^{-9} t^{6}-6.6248 \cdot 10^{-11} t^{7}+2.1460 \cdot 10^{-12} t^{8}-4.7663 \cdot 10^{-14} t^{9}+5.1985 \cdot 10^{-16} t^{10} \\
c(t)^{(10)}= & 0.1559-0.0045029 t+0.0001503 t^{2}+6.5375 \cdot 10^{-7} t^{3}-4.0130 \cdot 10^{-7} t^{4}+3.0252 \cdot 10^{-8} t^{5} \\
& -1.5877 \cdot 10^{-9} t^{6}+6.6325 \cdot 10^{-11} t^{7}-2.1592 \cdot 10^{-12} t^{8}+4.7935 \cdot 10^{-14} t^{9}-5.2211 \cdot 10^{-16} t^{10} \\
e(t)^{(10)}= & 0.1337+0.010998 t-0.0005127 t^{2}+1.5655 \cdot 10^{-5} t^{3}-3.5450 \cdot 10^{-7} t^{4}+6.2838 \cdot 10^{-9} t^{5} \\
& -8.7950 \cdot 10^{-11} t^{6}+9.0590 \cdot 10^{-13} t^{7}-4.7166 \cdot 10^{-15} t^{8}-2.9994 \cdot 10^{-17} t^{9}+5.4733 \cdot 10^{-19} t^{10} .
\end{aligned}
$$

Figs. 2-5 show the approximate solutions with 20 terms for $n(t), s(t), c(t)$ and $e(t)$ compared to the so-called exact solution of the smoking model. We see that HAM solutions are correct for a range of about 45 years for the evolution of 


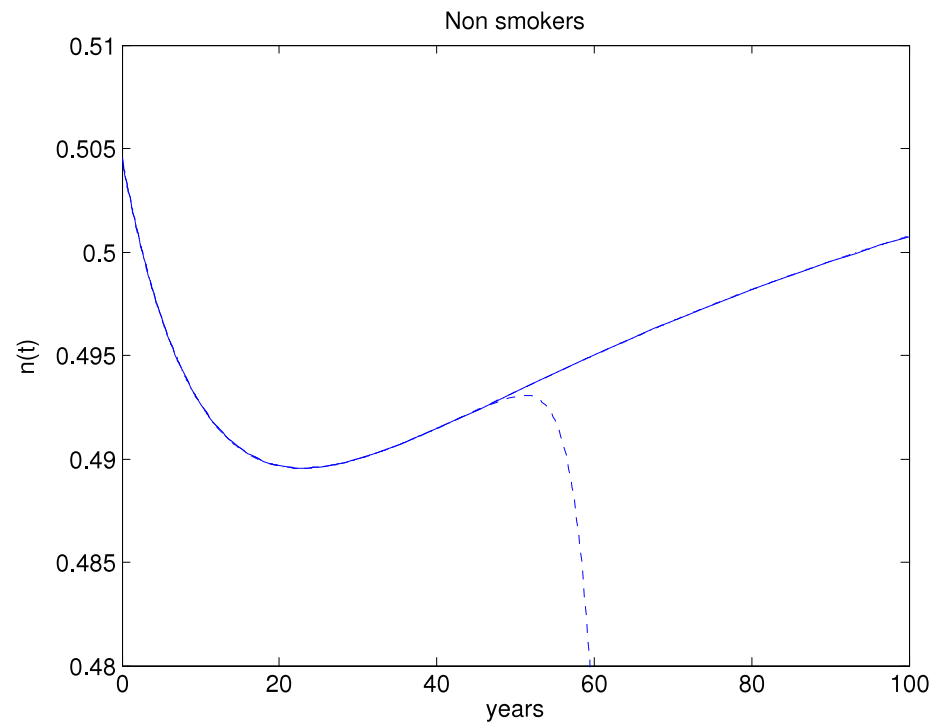

Fig. 7. Comparison between $[10,10]$ homotopy-Padé approximation (solid line), HAM solution with 20 terms (dashed line) and the exact solution (dash-dotted line) for $n(t)$.

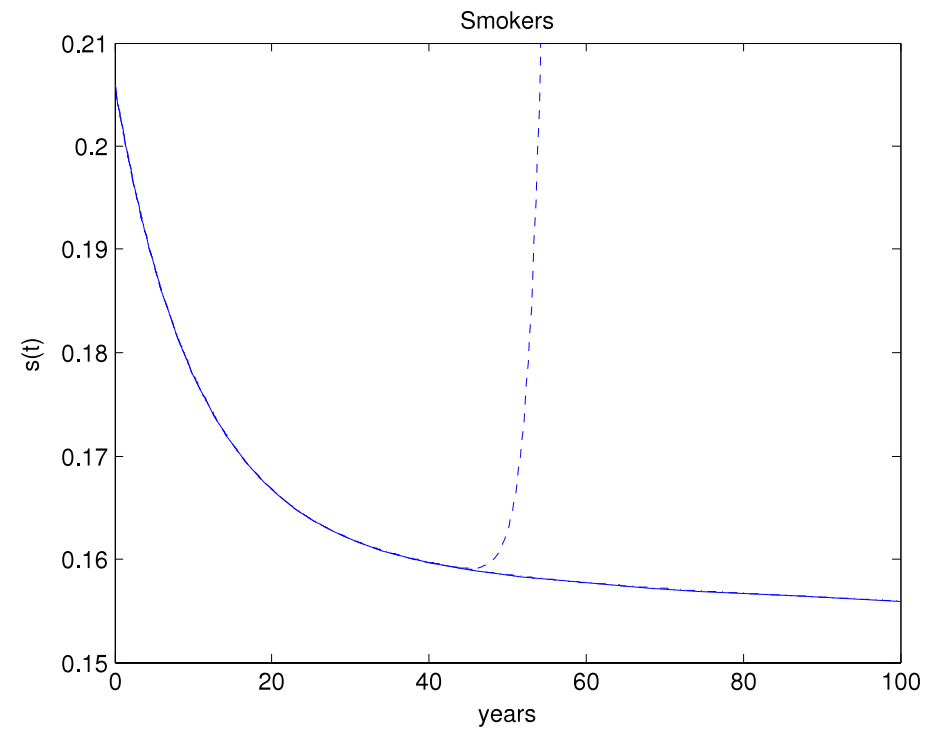

Fig. 8. Comparison between $[10,10]$ homotopy-Padé approximation (solid line), HAM solution with 20 terms (dashed line) and the exact solution (dash-dotted line) for $s(t)$

the prevalence of smoking. The exact solution is obtained by the fourth order Runge-Kutta method with a mesh width of $h=0.001$ years.

Fig. 6 shows HAM solutions for $s(t)$ with 5, 10, 15 and 20 terms to show the convergence to the exact solution. We see how, as the number of terms increases, the HAM solution is valid for a larger period of time. The HAM solutions for $n(t), c(t)$ and $e(t)$ have similar behaviour. For more about convergence of HAM solutions see [6].

\subsection{Homotopy-Padé technique results}

The homotopy-Padé technique was developed to accelerate the convergence of the HAM solution [6]. The idea is to apply the traditional Padé technique to Eq. (11) to get the $[m, m]$ Padé-approximant with respect to the parameter $q$ and then set $q=1$. The result that we obtain is a fraction with one polynomial of order $t^{m}$ in the numerator and the other polynomial of order $t^{m}$ in the denominator.

$$
[m, m] \rightarrow R_{m, m}=\frac{p_{0}+p_{1} t+p_{2} t^{2}+\cdots+p_{m} t^{m}}{1+s_{1} t+s_{2} t^{2}+\cdots+s_{m} t^{m}}
$$

Since we have calculated the HAM solution with 20 terms, we can obtain the $[10,10]$ homotopy-Padé approximation. Figs. 7-10 show the results of the [10, 10] homotopy-Padé approximations using the HAM solution with 20 terms presented in Figs. 2-5. 


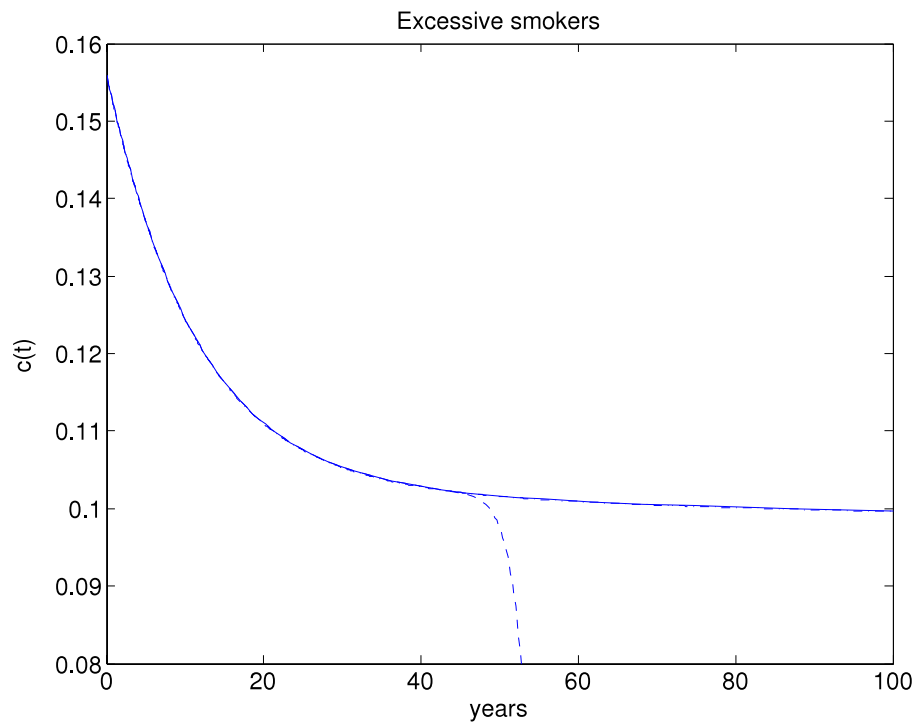

Fig. 9. Comparison between $[10,10]$ homotopy-Padé approximation (solid line), HAM solution with 20 terms (dashed line) and the exact solution (dash-dotted line) for $c(t)$.

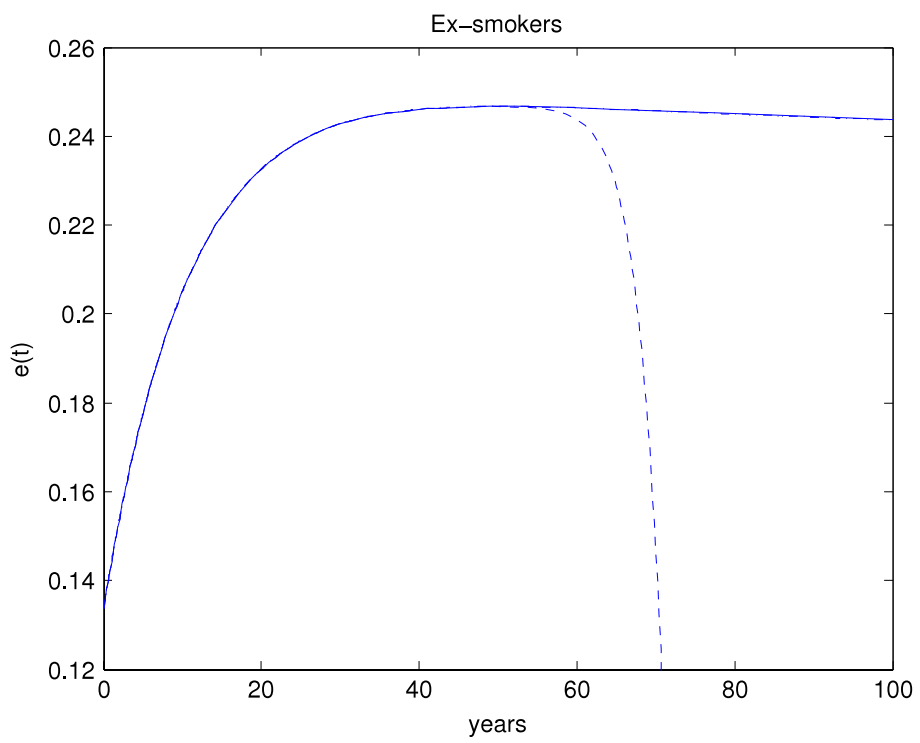

Fig. 10. Comparison between $[10,10]$ homotopy-Padé approximation (solid line), HAM solution with 20 terms (dashed line) and the exact solution (dash-dotted line) for $e(t)$. The Padé approximation is on the exact solution.

The convergence domain has been greatly enlarged. The HAM solution with 20 terms has a range of validity of 45 years. However, the $[10,10]$ homotopy-Padé solution is indistinguishable from the exact solution for a range of 100 years. In fact, its range of validity is about 180 years.

\section{Conclusions}

The analytical time power series that we have obtained for the four subpopulations proves that HAM is a very useful tool to solve this type of mathematical models for the spread of social habits.

Evaluating the optimal value of the control parameter we have obtained an analytical approximation for the solution with 20 terms which is valid for a range of time of more than 45 years. The range of validity of the approximation can be enlarged simply by adding more terms to the series. However, it is more efficient to enlarge the domain of convergence by means of the homotopy-Padé technique. We have been able to compute an analytical approximation which is valid for a period of more than 180 years.

Since the main purpose of this model is to predict the evolution of a social habit, we do not need a very large range of validity since social behaviours are continuously changing. In our smoking model, the parameters are assumed constant, and this fact cannot be valid for a very long period of time. This fact may make even an approximate solution with only five terms useful for practical purposes. Fig. 6 shows that a five term solution is a valid approximation for more than ten years. 


\section{References}

[1] F. Guerrero, F.J. Santonja, R.J. Villanueva, Analysing the effect of Spanish smoke-free legislation of year 2006: a new method to quantify its impact using a dynamic model, Int. J. Drug Policy 22 (2011) 247-251.

[2] E. Sánchez, R.J. Villanueva, F.J. Santonja, M. Rubio, Predicting cocaine consumption in Spain. A mathematical modeling approach, Drugs Edu. Prevent. Policy 18 (2)(2011) 105-108.

[3] F.J. Santonja, E. Sánchez, M. Rubio, J.L. Morera, Alcohol consumption in Spain and its economic costs: a mathematical modeling approach, Math. Comput. Modelling 52 (2010) 999-1003.

[4] F.J. Santonja, R.J. Villanueva, L. Jódar, G. González, Mathematical modeling of social obesity epidemic in the region of Valencia, Spain, Math. Comput. Model. Dyn. Syst. 16 (1) (2010) 23-34.

[5] J.D. Lambert, Numerical Methods for Ordinary Differential Systems: The Initial Value Problem, John Wiley and Sons, Chichester, UK, 1991.

[6] S.J. Liao, Beyond Perturbation: Introduction to the Homotopy Analysis Method, Chapman and Hall, CRC Press, Boca Raton, 2003.

[7] S.J. Liao, On the homotopy analysis method for nonlinear problems, Appl. Math. Comput. 174 (2004) 499-513.

[8] S.J. Liao, An explicit totally analytic approximation of Blasius viscous flow problems, Internat. J. Non-Linear Mech. 34 (1999) $759-778$.

[9] S.J. Liao, A new branch of solutions of boundary-layer flows over an impermeable stretched plate, Int. J. Heat Mass Transfer 48 (2005) $2529-3259$.

[10] S.J. Liao, An optimal homotopy-analysis approach for strongly nonlinear differential equations, Commun. Nonlinear Sci. Numer. Simul. 15 (2010) 2003-2016.

[11] S.J. Liao, Homotopy Analysis Method in Nonlinear Differential Equations, Springer \& Higher Education Press, Heidelberg, 2012.

[12] M. Ayub, A. Rasheed, T. Hayat, Exact flow of a third grade fluid past a porous plate using homotopy analysis method, Internat. J. Engrg. Sci. 41 (2003) 2091-2103.

[13] T. Hayat, M. Khan, Homotopy solutions for a generalized second-grade fluid past a porous plate, Nonlinear Dynam. 42 (2005) $395-405$.

[14] S. Abbasbandy, The application of homotopy analysis method to nonlinear equations arising in heat transfer, Phys. Lett. A 360 (2006) $109-113$.

[15] A. Sami, M.S.M. Noorani, I. Hashim, Solving systems of ODEs by homotopy analysis method, Commun. Nonlinear Sci. Numer. Simul. 13 (2008) 2060-2070.

[16] F. Awawdeh, A. Adawi, Z. Mustafa, Solutions of the SIR models of epidemics using HAM, Chaos Solitons Fractals 42 (2009) $3047-3052$.

[17] H. Khan, R.N. Mohapatra, K. Vajravelu, S.J. Liao, The explicit series solution of SIR and SIS epidemic models, Appl. Math. Comput. 215 (2009) 653-669. 